\title{
Ultrasound Dynamic Monitoring of The Inner Diameter of The Inferior Vena Cava To Guide The Application of Continuous Renal Replacement Therapy In Patients With Renal Failure Combined With Acute Heart Failure
}

\author{
Guang Yu \\ Second Affiliated Hospital of Nanchang University \\ Shaoyu Tao \\ Second Affiliated Hospital of Nanchang University \\ Yingzhi Jin \\ Second Affiliated Hospital of Nanchang University \\ Wanxia Li ( $\square$ wanxialidodo@sina.com ) \\ Second Affiliated Hospital of Nanchang University \\ Zanqun Hu \\ Second Affiliated Hospital of Nanchang University \\ Xiaowei Fang \\ Second Affiliated Hospital of Nanchang University
}

Research Article

Keywords: Inferior vena cava diameter and collapse with sniff, Continuous renal replacement therapy, Renal failure and acute heart failure

Posted Date: February 3rd, 2022

DOI: https://doi.org/10.21203/rs.3.rs-1261373/v1

License: (c) (i) This work is licensed under a Creative Commons Attribution 4.0 International License. Read Full License 


\section{Abstract}

Objective To explore the application value of bedside ultrasound dynamic monitoring of inferior vena cava diameter (IVCD) and collapse with sniff (IVCCI) to guide the application value of dehydration adjustment in continuous renal replacement therapy (CRRT) in patients with renal failure combined with acute heart failure. Methods We selected 90 patients with renal failure and acute heart failure who required continuous renal replacement therapy in the intensive care unit from January 2019 to June 2021. According to different blood volume assessment methods, patients were randomly divided into the ultrasound group, the experience group, and the control group. The inspection indicators of creatinine, $\mathrm{K}^{+}$, and NT-proBNP, the clinical indicators of improvement time of heart failure, time of continuous renal replacement therapy, use time of ventilator, ICU hospitalization time, and use time of vasopressor use and the incidence of adverse event were compared among the three groups.

Results There were no significant differences in creatinine, $\mathrm{K}^{+}$, and NT-proBNP between the three groups before and after treatment $(P>0.05)$. The improvement time of heart failure, time of continuous renal replacement therapy, and ICU hospitalization time in the ultrasound and experience group were lower than the control group, and the difference between the groups was statistically significant $(P<0.05)$. The use time of ventilator in the ultrasound and experience group was lower than the control group, there was a statistically significant difference between the ultrasound group and the control group $(P<0.05)$. The use time of vasopressor in the ultrasound group and the control group was lower than the experience group, and the difference between the groups was a statistically significant time $(P<0.05)$. The incidence of adverse events in the ultrasound group was lower than the experience group and the control group, and the difference between the groups was a statistically significant time $(P<0.05)$.

Conclusion Ultrasound dynamic monitoring of inferior vena cava diameter and collapse with sniff can more accurately assess the blood volume status, and provide a better guidance effect for the accurate dehydration of continuous renal replacement therapy and the rapid relief of heart failure symptoms in patients with renal failure and acute heart failure.

\section{Introduction}

Renal failure combined with acute heart failure is a clinical emergency and critical illness. It has the characteristics of rapid disease progression, a long hospital stay, and high mortality, which seriously threaten the life safety of patients.In clinical treatment, the main focus is to relieve the symptoms of heart failure, including treatments such as strengthening the heart, diuresis, and vasodilation. However, due to renal failure, the accumulated metabolites and water cannot be excreted through the kidneys. The effect of routine diuresis and vasodilation on hypertension and high-volume load is poor, while continuous renal replacement therapy (CRRT) can replace the damaged kidney by extracorporeal circulation blood purification and continuously remove the in vivo metabolites and excess water to reduce the preload and afterload of the heart and effectively improve the symptoms and condition of patients with heart failure.However, various complications occur from time to time during the clinical application of CRRT, among which hypotension is one of the main complications. Some studies have shown that the degree of blood volume decline is an important cause of blood pressure changes in CRRT treatment. Excessive and rapid dehydration leads to dehydration much greater than the reflux of tissue fluid, then the insufficient effective blood volume leads to hypotension. However, how to correctly assess the blood volume status of patients during CRRT treatment and formulate a reasonable dehydration plan is a difficult problem faced by clinicians. In recent years, ultrasound measurement of inferior vena cava diameter (IVCD) and variability (Inferior vena cava diameter and collapse with sniff, IVCCI) has been widely used in volume assessment due to its intuitive, accurate, non-invasive, and repeatable advantages. Many studies are suggesting that the inferior vena cava diameter can be used as a guiding method for evaluating the blood volume status of patients [4-6], but there are still few reports on the application of CRRT in patients with renal failure combined with acute heart failure. Therefore, this study aims to explore the application value of bedside ultrasound dynamic monitoring of IVCD and IVCCI in guiding the adjustment of dehydration in the treatment of CRRT in patients with renal failure combined with acute heart failure. The report is as follows.

\section{Materials And Methods}

The research protocol was approved by the Medical Ethics Committee of the Second Affiliated Hospital of Nanchang University, and informed consent was obtained from all individual participants or their legal guardian included in the study. All methods were carried out in accordance with relevant guidelines and regulations. Data collection began October 2019 and concluded October 2021.

\subsection{Study patients}


We selected 90 patients with renal failure combined with acute heart failure who were admitted to the ICU of our hospital from January 2019 to June 2021 and required CRRT treatment, including 62 males and 28 females, with an average age of (68.23 \pm 11.41$)$ years. Inclusion criteria: (1) meet the diagnostic criteria for renal failure combined with acute heart failure, refer to the KDIGO diagnostic criteria for the diagnosis of renal failure, refer to guidelines for the primary diagnosis and treatment of acute heart failure (2019) (2) agree to CRRT treatment; (3) age $\geq 18$ years old and $\leq 80$ years old. Exclusion criteria: (1) suffering from malignant tumors or psychiatric diseases; (2) suffering from congenital heart disease, hypertrophic cardiomyopathy, pulmonary hypertension; (3) patients with abnormal coagulation function or diseases such as intracerebral hemorrhage, visceral hemorrhage, and gastrointestinal hemorrhage in recent 3 months, with heparin anticoagulant contraindications;(4) CRRT treatment time $\leq 12$ hours; (5) patients whose inferior vena cava cannot be detected by ultrasound and data cannot be collected; (6) cardiogenic shock, cardiac ejection fraction measured by color ultrasound $\leq$ $50 \%$.Using the random number table method, 90 patients were randomly divided into three groups: ultrasound group, experience group, and control group with 30 cases in each group. The differences in gender, age, and APACHE II score of the three groups were not statistically significant and comparable, as shown in Table 1.

\subsection{Methods}

1.2.1 Ultrasound monitoring IVCD and IVCCI evaluation group.

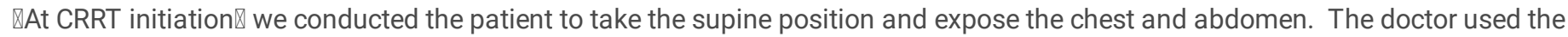
$3.5 \mathrm{MHz}$ convex array probe of Mindray M7 portable color Doppler ultrasound to measure the section of the inferior vena cava inferior to the xiphoid process. At a distance of $2.0 \mathrm{~cm}$ from the right heart of the inferior vena cava, multiple breathing cycles were collected with Mmode ultrasound, and the maximum end-inspiratory diameter (IVCDmax) and the minimum end-expiratory diameter (IVCDmin) were measured at the same time. IVCD takes the maximum inner diameter of the end of inspiration (IVCDmax), and IVCCI is calculated according to the formula (IVCDmax-IVCDmin)/IVCDmax $100 \%$. All ultrasound examinations were completed by qualified critical care physicians who had received ultrasound training. Each index was measured 3 times and the average value was taken; the internal diameter and variability of inferior vena cava were measured by ultrasound every 4 hours until CRRT discontinuation.

QThe volume status is evaluated according to the practical guidelines of the British Society of Echocardiography[7], IVCD $\leq 2.1 \mathrm{~cm}$ with IVCCI $\$ 50 \%$, showing a low volume state; and IVCD $\leq 2.1 \mathrm{~cm}$ with IVCCI $85 \%$, or IVCD $\nabla 2.1 \mathrm{~cm}$ with IVCCI $₫ 50 \%$, showing a state of volume balance; when IVCD $₫ 2.1 \mathrm{~cm}$ with IVCCI $₫ 50 \%$, it is a state of high volume; $邓 I$ IV IVCD and IVCCI indicate a high volume state, adjust the 4-hour target dehydration volume to $1000 \mathrm{ml}$; if they indicate a state of volume balance, adjust the 4-hour target dehydration volume to $500 \mathrm{ml}$; if the low-volume state is prompted, adjust the 4-hour target dehydration volume to $0 \mathrm{ml}$. (CRRT dehydration volume in 4 hours $=4$-hour target dehydration volume + fluid volume in 4 hours-urine volume in 4 hours).

\subsubsection{General empirical evaluation group.}

QRecord the heart rate, mean arterial pressure, central venous pressure, wet rales of the lungs, and other data after CRRT on the machine, and adjust the amount of dehydration according to the general empirical method scoring table (see Table 2). $₫$ Scoring is performed every 4 hours from the beginning of CRRT to getting off the machine. For a score of 8-11 points, adjust the 4-hour target dehydration volume to $1000 \mathrm{ml}$; for a score of 4-7 points, adjust the 4-hour target dehydration volume to $500 \mathrm{ml}$; for a score of 0-3 points, adjust the 4-hour target dehydration volume to $0 \mathrm{ml}$. (CRRT dehydration volume in 4 hours $=4$-hour target dehydration volume + fluid volume in 4 hours-urine volume in 4 hours).

\section{Table 1 Characteristics of the study population}

\begin{tabular}{|lllll|}
\hline \multicolumn{1}{|c}{ Group } & Cases & $\mathrm{M} / \mathrm{F}$ & Age & APACHE II \\
\hline Ultrasound group & 30 & $21 / 9$ & $67.57 \pm 12.97$ & $20.17 \pm 6.87$ \\
\hline Experience group & 29 & $21 / 8$ & $69.59 \pm 10.60$ & $19.28 \pm 5.10$ \\
\hline Control group & 29 & $18 / 11$ & $68.45 \pm 10.64$ & $19.17 \pm 4.05$ \\
$\chi^{2} / F$ & & 0.79 & 0.23 & 0.29 \\
$P$ & & 0.68 & 0.80 & 0.75 \\
\hline
\end{tabular}

Note: We changed one patient's anticoagulation mode who had had gastrointestinal bleeding in the experience group, and one patient in the control group gave up treatment, all of which have been canceled and not included in the statistical analysis. 


\begin{tabular}{|clllc|}
\hline Score & 3 points & 2 points & 1 points & 0 points \\
\hline HR & $\geq 180$ & $140-179$ & $110-139$ & $50-109$ \\
\hline MAP & $\geq 160$ & $130-159$ & $110-129$ & $65-109$ \\
\hline CVP & $\geq 16$ & $12.1-15.9$ & $5-12$ & $2.5-4.9$ \\
\hline PMR & & more than $50 \%$ of both lung fields & less than $50 \%$ of both lung field & not seen \\
\hline
\end{tabular}

$\mathrm{HR}=$ heart rate; $\mathrm{MAP}=$ Mean arterial pressure; $\mathrm{CVP}=$ central venous pressure; $\mathrm{PMR}=$ pulmonary moist rales.

\subsubsection{Control group}

From the beginning of CRRT to getting off the machine区the target dehydration volume is constant at $100 \mathrm{ml} / \mathrm{h}$, and no fluid evaluation and adjustment are performed during the treatment process. (CRRT dehydration volume in 4 hours $=4$-hour target dehydration volume + fluid volume in 4 hours-urine volume in 4 hours).

\subsubsection{Homogeneous treatment.}

QIn addition to the above-mentioned target dehydration adjustment experimental measures, the three groups of patients all adopted homogeneous treatment. For example, primary disease treatment, anti-infection scheme, airway management, mechanically assisted ventilation strategy, maintaining water and electrolyte balance $(4.0 \mathrm{mmol} / \mathrm{l}<\mathrm{K}+<5.3 \mathrm{mmol} / \mathrm{l})$, drug treatment scheme, colloidal liquid supplements such as albumin (maintaining albumin $>3.5 \mathrm{~g} / \mathrm{I}$ ) and nutritional support, etc. $\otimes$ The three groups of patients were treated with the same brand and model of blood purification machine (PrismaFlex system) for CRRT treatment (CVVHD mode). They were all treated with extracorporeal heparin for local anticoagulation and protamine neutralization and adjusted the dosage of heparin and protamine according to the four coagulation items (APTT maintained at 1 of the normal value 1-1.5 times). In CRRT treatment, blood flow was 150$200 \mathrm{ml} / \mathrm{min}$ and dialysate flow was $2000 \mathrm{ml} / \mathrm{h}$. (Dialysate formula: NS $2000 \mathrm{ml}$, sterile water for injection $1000 \mathrm{ml}, 50 \% \mathrm{GS} 10 \mathrm{ml}, 10 \% \mathrm{NS}$ $20 \mathrm{ml}$, MgSO $2.5 \mathrm{ml}, 10 \% \mathrm{KCL} 7.5 \mathrm{ml}, \mathrm{NaHCO} 345 \mathrm{ml}$, peripherally pumped $\mathrm{CaCl} 210 \mathrm{ml} / \mathrm{h}$ ); $邓$ When the patient developed hypotension, we would immediately suspend dehydration and replenish fluids and give vasoactive drugs if deemed necessary (including norepinephrine, dopamine, etc.) to maintain the patient's MAP above $65 \mathrm{mmHg}$.

\subsection{Observation indicators}

QThe test indexes of the three groups before and after CRRT treatment for 24 hours: blood creatinine, blood potassium, and NTproBNP $\triangle T$ Test index of the three groups of patients before and after CRRT treatment for 24 hours: serum creatinine, serum potassium, NTproBNP; 『Clinical indicators of the three groups of patients during ICU hospitalization: heart failure improvement time, CRRT treatment time, ventilator use time, ICU hospitalization time, use time of vaso- pressor; $₫ D$ ata on the incidence of adverse events (hypotension, arrhythmia, delirium) during CRRT treatment in the three groups.

\subsection{Assessment criteria of heart failure improvement}

\Symptom improvement: chest tightness was significantly improved, and cough and expectoration were significantly reduced; $\nabla$ Physical signs improved: wet rales in the lungs decreased significantly; $\$ Monitoring indicators improved: heart rate, respiratory rate, mean arterial pressure and central venous pressure decreased significantly. The improvement of the above three indexes is rated as the improvement of heart failure symptoms, which is objectively evaluated once every hour.

\subsection{Statistical methods.}

The analysis was performed using SPSS22.0 software. Continuous variables were expressed as mean \pm standard deviation, and the comparison of means was performed using a t test. The categorical data were expressed as frequencies and percentages. A $p$ value of $<0.05$ was considered to be statistically significant.

\section{Results}

2.1 Comparison of test indexes of three groups before and after CRRT treatment for $\mathbf{2 4}$ hours. 
The serum creatinine, serum potassium, and NT-proBNP indexes of the three groups of patients decreased after 24 hours of CRRT treatment. Differences within the groups were statistically significant (Pख0.05) and there was no significant difference in pairwise comparison among the 3 groups (P凶0.05), see Table 3.

\section{2 clinical indicators}

The improvement time of heart failure, CRRT treatment time, and ICU stay in the ultrasound group and the experience group were significantly lower than those of the control group, and the difference between the groups was statistically significant (P凶0.05), while there was no significant difference in the above indicators between the ultrasound group and the experience group (Pख0.05). The use time of

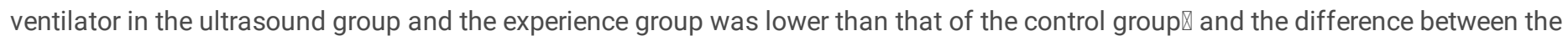
ultrasound group and the control group was statistically significant $(P \otimes 0.05)$, while there was no significant difference in experience group and the control group as well as the experience group and the ultrasound group (P凶0.05). The ultrasound group and the control group used vasopressor for less time than the experience group, and the difference between the groups was statistically significant (Pख0.05), while the ultrasound group and the control group had no significant difference (P凶0.05). see Table 4.

\subsection{Comparison of incidence of adverse events}

The incidence of adverse events in the ultrasound group was significantly lower than that of the experience group and the control group, and the difference was statistically significant (Pख0.05), but the difference between the experience group and the control group was not statistically significant $(\mathrm{P} \otimes 0.05)$, see Table 5 .

Table 3 Comparison of creatinine, $\mathrm{K}^{+}$, and NT-proBNP of three groups before and after CRRT treatment for 24h

\begin{tabular}{|llllllll|}
\hline Group & Number & before & & & after & & \\
& $\begin{array}{l}\text { of } \\
\text { cases }\end{array}$ & Creatinine & $\mathrm{K}^{+}$ & NT-proBNP & Creatinine & $\mathrm{K}^{+}$ & NT-proBNP \\
\cline { 3 - 8 } & & & & & & & \\
$\begin{array}{l}\text { Ultrasound } \\
\text { group }\end{array}$ & 30 & $817.08 \pm 162.77$ & $5.67 \pm 0.64$ & $23059.48 \pm 9964.45$ & $410.97 \pm 95.90^{\#}$ & $4.08 \pm 0.43^{\#}$ & $11367.24 \pm 4921.83^{\#}$ \\
\hline $\begin{array}{l}\text { Experience } \\
\text { group }\end{array}$ & 29 & $803.87 \pm 140.48$ & $5.59 \pm 0.83$ & $24001.42 \pm 9850.54$ & $400.15 \pm 87.14^{\#}$ & $4.11 \pm 0.31^{\#}$ & $14414.07 \pm 6989.10^{\#}$ \\
\hline $\begin{array}{l}\text { Control } \\
\text { group }\end{array}$ & 29 & $827.25 \pm 151.29$ & $5.66 \pm 0.75$ & $24987.59 \pm 11492.37$ & $422.60 \pm 93.40^{\#}$ & $4.14 \pm 0.32^{\#}$ & $13160.29 \pm 7501.93^{\#}$ \\
\hline$t$ & & 0.17 & 0.09 & 0.25 & 0.43 & 0.22 & 1.61 \\
\hline$P$ & 0.842 & 0.91 & 0.78 & 0.65 & 0.80 & 0.21 \\
\hline
\end{tabular}

\# indicates significant difference compared with before》 $P<0.05$.

Table 4 Comparison of three groups of clinical indicators

\begin{tabular}{|c|c|c|c|c|c|c|}
\hline Group & $\begin{array}{l}\text { Number } \\
\text { of } \\
\text { cases }\end{array}$ & 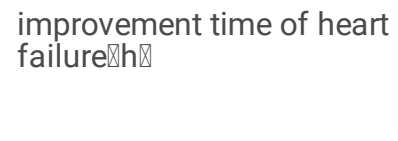 & $\begin{array}{l}\text { CRRT treatment } \\
\text { time } ₫ \mathrm{~h} \rrbracket\end{array}$ & $\begin{array}{l}\text { Ventilator use } \\
\text { time(h) }\end{array}$ & $\begin{array}{l}\text { ICU } \\
\text { stay } \llbracket \mathrm{d} \rrbracket\end{array}$ & $\begin{array}{l}\text { Vasopressor } \\
\text { use time『h }\end{array}$ \\
\hline $\begin{array}{l}\text { Ultrasound } \\
\text { group }\end{array}$ & 30 & $3.00 \pm 2.51$ & $36.80 \pm 15.27$ & $10.80 \pm 19.88$ & $4.43 \pm 2.40$ & $2.40 \pm 5.91^{*}$ \\
\hline $\begin{array}{l}\text { Experience } \\
\text { group }\end{array}$ & 29 & $3.17 \pm 2.04$ & $38.48 \pm 19.09$ & $23.21 \pm 50.43$ & $4.69 \pm 2.75$ & $10.83 \pm 10.74$ \\
\hline $\begin{array}{l}\text { Control } \\
\text { group }\end{array}$ & 29 & $5.17 \pm 3.58^{\# \star}$ & $73.00 \pm 33.93^{\#^{*}}$ & $31.86 \pm 34.91^{\#}$ & $6.17 \pm 2.89^{\#^{*}}$ & $3.90 \pm 7.38^{*}$ \\
\hline$t$ & & 5.50 & 21.05 & 2.41 & 3.57 & 8.72 \\
\hline$P$ & & $P<0.05$ & $P<0.05$ & 0.10 & $P<0.05$ & $P<0.05$ \\
\hline
\end{tabular}

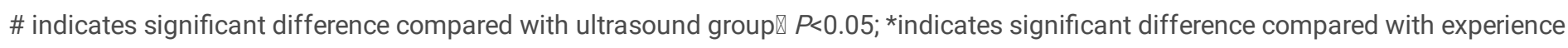
group $\mathbb{P}<0.05$. 


\begin{tabular}{|lll|}
\hline Group & Number of cases & incidence of adverse events \\
\hline Ultrasound group & 30 & $16.7 \%$ \\
\hline Experience group & 29 & $55.2 \%^{\#}$ \\
\hline Control group & 29 & $48.3 \%^{\#}$ \\
$x^{2}$ & & 10.43 \\
$P$ & & $P<0.05$ \\
\hline
\end{tabular}

\# indicates significant difference compared with ultrasound group $₫<<0.05$.

\section{Discussion}

Renal failure combined with acute heart failure is a complex pathophysiological process caused by various factors leading to renal function damage. Metabolites and excessive water in the body cannot be excreted through the kidneys, resulting in the accumulation of metabolites and large amounts of fluid in the body, increasing blood volume, interstitial edema, causing the heart pre-and post-load increasing, which in turn produces acute heart failure.

Renal failure and heart failure affect and aggravate each other, forming a vicious circle, which ultimately leads to a rapid deterioration of heart and kidney function and seriously threatens the lives of patients. In clinical practice, CRRT is often used to replace the kidneys to remove excess water and metabolites from the body and improve the patient's condition. However, it is still difficult to achieve a rapid, efficient and safe relief of the symptoms of heart failure in patients. Therefore, how to correctly assess the blood volume status is critical for guiding and adjusting CRRT dehydration.

At present, the mainstream methods for evaluating blood volume at home and abroad are as follows: pulmonary artery catheterization, pulse indicating continuous cardiac output, transesophageal echocardiography, bioimpedance method, etc. [8-11]. These technologies have their own advantages, but there are many limitations. Many clinicians are still keen to assess the blood volume of patients through general empirical methods during CRRT treatment, such as estimating the patient's dry weight, whether there are pulmonary moist rales, whether there is acral and facial edema and changes in vital signs according to experience. Although these methods are simple and easy, they have poor reliability and cannot meet the requirements of rapid, dynamic, accurate and noninvasive clinical evaluation of blood volume changes in patients with CRRT.

In this study, the ultrasonic method and empirical method were used to evaluate the blood volume of patients and compared with the control group. The results show that, the serum creatinine, serum potassium, and NT-proBNP indexes of the three groups of patients decreased after 24 hours of CRRT treatment. Differences within the groups were statistically significant and there was no significant difference in pairwise comparison among the 3 groups This confirms that CRRT dehydration treatment can effectively help patients with renal failure and acute heart failure to remove excess water, metabolic waste, maintain electrolyte balance and improve the patient's heart function. Different blood volume assessment methods did not affect the clearance efficiency of creatinine and serum potassium during the initial treatment, and no significant effect was found on the NT-proBNP index.

Further research found that the improvement time of heart failure, CRRT treatment time and ICU stay in the ultrasound group and the experience group were significantly lower than those of the control group. Compared with the control group, the ultrasound group had significantly lower ventilator use time, and the difference was statistically significant. The above results show that compared with the control group without volume assessment, the ultrasound group and experience group with volume assessment can improve the symptoms of heart failure faster, shorten the time of CRRT treatment and ICU hospitalization.

It is confirmed that timely dynamic volume assessment during CRRT treatment has better clinical value to guide the adjustment of dehydration in patients with renal failure combined with acute heart failure. In addition, the use time of the ventilator in the ultrasound group was also significantly shortened, further indicating that the use of ultrasound volume assessment to adjust the amount of dehydration has a more obvious clinical effect. 
In the comparison of the use time of vasopressor, and the incidence of adverse events $邓$ hypotension, arrhythmia, delirium $\rrbracket$, we found that the use time of booster drugs in the ultrasound group and the control group was significantly less than that of the experience group, and

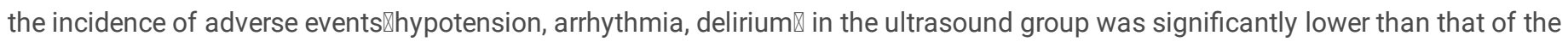
experience group and the control group. After analyzing the above results, we believe that there are the following reasons. First, although the empirical volume assessment method has a certain value in the assessment of high volume load, such as quickly improving the symptoms of heart failure in patients, shortening the CRRT treatment time and the length of ICU stay, the accuracy of the assessment of insufficient volume remains to be discussed. This may be related to the patient's acute onset, chest tightness, palpitation, nervousness, and other stress factors that lead to sympathetic nerve excitement, which further causes increased heart rate and blood pressure, leading to the illusion of high volume load in the empirical volume assessment results. During the CRRT treatment, the amount of dehydration is too fast and too high resulting in the effective blood volume being insufficient, which increases the risk of CRRT-related hypotension and the use time of vasopressor. Second, the dehydration of patients in the control group was slow and uniform. Although the use time of vasopressor was shorter, heart failure symptoms were relieved more slowly, the CRRT treatment time significantly increased, the length of stay in ICU prolonged, and the incidence of adverse events such as arrhythmia and delirium increased. In contrast, in the ultrasound group, not only did the symptoms of heart failure improve rapidly but also the CRRT treatment time, ICU hospitalization time, and ventilator use time were significantly shortened. More importantly, the incidence of CRRT related hypotension and the use time of pressor drugs were less and the incidence of adverse events was significantly reduced and the effect was better.

It is worth mentioning that this study still has its limitations (small sample size, short time and single-center). Therefore, in the future, it is still necessary to further carry out multi-center and large sample experiments in order to draw more real and effective conclusions and provide a better basis for clinicians to guide the adjustment of CRRT dehydration.

In conclusion, due to the rapid progress of patients with renal failure combined with acute heart failure, the evaluation of blood volume needs to be more intuitive and accurate. Ultrasonic dynamic monitoring IVCD and ivcci can provide accurate guidance for CRRT dehydration adjustment in patients with renal failure combined with acute heart failure in a more accurate and timely manner. It can

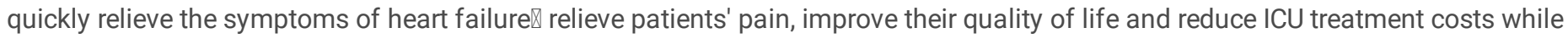
reducing the incidence of adverse events, so it has good social and economic benefits.

\section{Declarations}

\section{Funding}

This work was supported by the Health Commission Science and Technology Project of Jiangxi Province (No.202210624).

\section{Competing interests}

The authors declare no competing interests.

\section{Author contributions}

Guang Yu and Shaoyu Tao contributed equally to this work. Guang Y and Shaoyu Tao designed the study. Guang Yu, Yingzhi Jin and Zanqun Hu conducted the data collection under supervision of Wanxia Li. Guang Yu conducted the data analyses under supervision of Wanxia Li. Guang Yu and Shaoyu Tao wrote the manuscript and Xiaowei Fang and Wanxia Li commented the manuscript. Yingzhi Jin translated manuscript. All authors have read and approved the final version of the manuscript.

\section{Data availability}

The datasets used and/or analysed during the current study available from the corresponding author on request.

\section{References}

[1] Sigwalt F, Bouteleux A, Dambricourt F,et al. Clinical Complications of Continuous Renal Replacement Therapy[J]. Contrib Nephrol, 2018, 194:109-117.

[2] Douvris A, Zeid K, Hiremath S, et al. Mechanisms for hemodynamic instability related to renal replacement therapy: a narrative review[J]. Intensive Care Med,2019, 45(10): 1333-1346. 
[3]Reeves PB, Mc Causland FR. Mechanisms, Clinical Implications, and Treatment of Intradialytic Hypotension[J]. Clin J Am Soc Nephrol, 2018, 13(8):1297-1303.

[4] Vaish H, Kumar V, Anand R, et al. The Correlation Between Inferior Vena Cava Diameter Measured by Ultrasonography and Central Venous Pressure[J]. Indian J Pediatr, 2017, 84(10):757-762.

[5] Zhang J, Critchley LA. Inferior Vena Cava Ultrasonography before General Anesthesia Can Predict Hypotension after Induction[J]. Anesthesiology, 2016, 124(3): 580-589.

[6] Bortolotti P, Colling D, Colas V, et al. Respiratory changes of the inferior vena cava diameter predict fluid responsiveness in spontaneously breathing patients with cardiac arrhythmias[J]. Ann Intensive Care, 2018,8(1): 79.

[7] Zaidi A, Knight DS, Augustine DX, et al. Echocardiographic assessment of the right heart in adults: a practical guideline from the British Society of Echocardiography[J]. Echo Res Pract, 2020, 7(1): G19-G41.

[8] De Backer D. Vincent JL. The pulmonary artery catheter: is it still alive? [J].Curr Opin Crit Care 2018, 24(3): $204-208$.

[9] Monnet X, Teboul JL. Transpulmonary thermodilution: advantages and limits[J].Crit Care, 2017, $21(1): 147$.

[10] Boissier F, Bagate F, Mekontso Dessap A. Hemodynamic monitoring using trans esophageal echocardiography in patients with shock[J]. Ann Transl Med 2020, 8(12): 791.

[11] Sangkum L, Liu GL, Yu L, et al. Minimally invasive or noninvasive cardiac output measurement: an update[J]. J Anesth 2016, 30(3): 461-480.

\section{Supplementary Files}

This is a list of supplementary files associated with this preprint. Click to download.

- RawData.xIsx 